Pacific Journal of Mathematics

INVARIANT SUBSPACES IN THE POLYDISK

OM P. Agrawal, Douglas Napier Clam
and Ronald George Douglas 


\title{
INVARIANT SUBSPACES IN THE POLYDISK
}

\author{
O. P. Agrawal, D. N. Clark and R. G. Douglas
}

This note is a study of unitary equivalence of invariant subspaces of $H^{2}$ of the polydisk. By definition, this means joint unitary equivalence of the shift operators restricted to the invariant subspaces.

In one variable, all invariant subspaces are unitarily equivalent and all can be represented as inner functions times $H^{2}$. In several variables, our results suggest that unitary equivalence and multiplication by inner functions are again related. For example, all invariant subspaces of a given invariant subspace $\mathscr{M}$ which are unitarily equivalent to $\mathscr{M}$ are $\varphi \mathscr{M}$, for $\varphi$ inner; and all invariant subspaces unitarily equivalent to an invariant subspace $\mathscr{M}$ of finite codimension are $\varphi \mathscr{M}$. In particular, two invariant subspaces of finite codimension are unitarily equivalent if and only if they are equal.

0. The classic paper of Beurling [4] led to a spate of research in operator theory, $H^{p}$ theory, and other areas, which continues to the present. Although Beurling's answer to the problem of spectral synthesis was negative, his explicit characterization of the invariant subspaces of the unilateral shift, in terms of the inner-outer factorization of analytic functions has had a major impact. Since the Hardy space for the unit disk is the primary nontrivial example for so many different areas, it is not surprizing that this characterization has proved so important.

Almost everyone who has thought about this topic must have considered the corresponding problem for $\mathrm{H}^{2}$ of the polydisk. Although the existence of inner functions in this context is obvious (in contrast with the case of the ball), one quickly sees that a Beurling-like characterization is not possible. Most results on this problem have gone unpublished [10], except for the book of Rudin [11], and many results are counterexamples. One exception is a characterization, by Ahern and the second author, of invariant subspaces having finite codimension, as the closure of the ideals in $C\left[z_{1}, \ldots, z_{N}\right]$ of finite codimension; [2]. In this note, we eschew the goal of characterizing all invariant subspaces in the polydisk and instead investigate equivalence of invariant subspaces in $N$ variables, under joint unitary equivalence of the restrictions of the $N$ coordinate shifts. Put another way, we shall consider the problem of characterizing the (inequivalent) submodules of the Hardy space over the polydisk algebra. The existence of nonunitarily equivalent submodules is directly related to the 
failure of inner functions to characterize invariant subspaces; [7]. We believe that this may be a worthwhile approach to this problem. A discussion of this point of view has been published by $\mathrm{M}$. Cowen and the third author in [8].

The first result on nonunitarily equivalent invariant subspaces is due to Berger, Coburn and Lebow [3], who considered the restrictions of multiplication by the coordinate functions to the invariant subspaces obtained as the closure of certain ideals in $\mathbf{C}\left[z_{1}, z_{2}\right]$ having the origin as zero set. By applying their results on commuting isometries, they showed that different ideals yield inequivalent invariant subspaces. In [7], Cowen and the third author reproved these results using their complex geometric approach to operator theory. In his Dissertation [1], the first author extended these methods to cover the invariant subspaces defined by most ideals in $\mathbf{C}\left[z_{1}, z_{2}\right]$ with zero set consisting of an arbitrary point in the bidisk. This proof is indicated in $\$ 5$ below. The results of [1] led us to realize that all ideals in $\mathbf{C}\left[z_{1}, z_{2}, \ldots, z_{N}\right]$ of finite codimension define nonunitarily equivalent invariant subspaces of $H^{2}$. We present two distinct proofs of this result in $\S \S 3$ and 4 , along with a number of questions. The different proofs generalize in different directions.

1. Let $\mathbf{Z}$ denote the integers, $\mathbf{Z}_{+}$the nonnegative integers and $N$ a fixed positive integer greater than 1 . For a subset $A$ of $\mathbf{Z}^{N}$, we identify $l^{2}(A)$ with the Hilbert space of Fourier series

$$
f\left(e^{i t_{1}}, \ldots, e^{i t_{N}}\right) \sim \sum_{\alpha \in A} a_{\alpha} e^{i \alpha t},
$$

with $\|f\|^{2}=\sum_{\alpha \in A}\left|a_{\alpha}\right|^{2}<\infty$, where $e^{i \alpha t}=e^{i \alpha_{1} t_{1}} \cdots e^{i \alpha_{N} t_{N}}$. Such a function $f$ can be viewed as defined on the $N$ torus $\mathbf{T}^{N}$; this correspondence defines the usual isometrical isomorphism between $l^{2}\left(\mathbf{Z}^{N}\right)$ and $L^{2}\left(\mathbf{T}^{N}\right)$. The subspace $l^{2}\left(\mathbf{Z}_{+}^{N}\right)$ corresponds to the Hardy space $H^{2}\left(\mathbf{T}^{N}\right)$.

Each function $\varphi$ in $L^{\infty}\left(\mathbf{T}^{N}\right)$ determines on $L^{2}\left(\mathbf{T}^{N}\right)$ a multiplication operator $M_{\varphi}$, defined by

$$
M_{\varphi} f=\varphi f .
$$

When $\varphi \in H^{\infty}\left(\mathbf{T}^{N}\right)$, the operator $M_{\varphi}$ leaves $H^{2}\left(\mathbf{T}^{N}\right)$ invariant, and by an invariant subspace of $H^{2}\left(\mathbf{T}^{N}\right)$, we mean a closed subspace of $H^{2}\left(\mathbf{T}^{N}\right)$, invariant under $M_{\varphi}$ for $\varphi \in H^{\infty}$; or, equivalently, invariant under the shifts $S_{1}=M e^{i t_{1}}, \ldots, S_{N}=M e^{i t_{N}}$. Two invariant subspaces $\mathscr{M}_{1}$ and $\mathscr{M}_{2}$ are said to be unitarily equivalent if there is a unitary operator $U$ : $\mathscr{M}_{1} \rightarrow \mathscr{M}_{2}$ such that $U(\varphi f)=\varphi(U f)$, for $\varphi \in H^{\infty}\left(\mathbf{T}^{N}\right)$ and $f \in \mathscr{M}_{1}$. This is equivalent to joint unitary equivalence of the restrictions $\left.S_{1}\right|_{\mathscr{M}}, \ldots,\left.S_{N}\right|_{\mathscr{M}_{j}}$, for $j=1,2$. 
The above notion of equivalence can also be viewed as equivalence of submodules of $H^{2}\left(\mathbf{T}^{N}\right)$ over the polydisk algebra $A\left(\mathbf{D}^{N}\right)$ (D the open unit disk). Here we are using submodule to mean closed subspace of $H^{2}\left(\mathbf{T}^{N}\right)$, invariant under the action of $A\left(\mathbf{D}^{N}\right)$. The natural notion of unitary equivalence of modules then becomes unitary equivalence of invariant subspaces, as defined above.

We begin with a lemma which exhibits the form a unitary equivalence of invariant subspaces must have.

LEMMA 1. If $\mathscr{M}_{1}$ and $\mathscr{M}_{2}$ are invariant subspaces of $H^{2}\left(\mathbf{T}^{N}\right)$ and if $U$ : $\mathscr{M}_{1} \rightarrow \mathscr{M}_{2}$ is a unitary operator making the restricted shifts jointly unitarily equivalent

$$
\left.U S_{n}\right|_{\mathscr{M}_{1}}=\left.S_{n}\right|_{\mathscr{M}_{2}} U, \quad n=1, \ldots, N,
$$

then there exists a unimodular function $\varphi \in L^{\infty}\left(\mathbf{T}^{N}\right)$, such that

$$
U f=\varphi f, \quad f \in \mathscr{M}_{1} .
$$

Proof. Since $\mathscr{M}_{1}$ and $\mathscr{M}_{2}$ are invariant subspaces, the restrictions of $S_{1}, \ldots, S_{N}$ to $\mathscr{M}_{1}$ and $\mathscr{M}_{2}$ are commuting $N$-tuples of isometries, the minimal unitary extensions of which are both $S_{1}, \ldots, S_{N}$ on $L^{2}\left(\mathbf{T}^{N}\right)$ (the proof is the same as that in the one variable case; cf. [5, Theorem 12]). Now a straightforward application of a corollary in [9] shows that $U$ can be extended to a unitary operator $W$ on $L^{2}\left(\mathbf{T}^{N}\right)$ that commutes with the multiplication operators $\left\{S_{n}\right\}_{n=1}^{N}$. Setting $\varphi=W 1$, we obtain, for $f \sim$ $\sum a_{\alpha} e^{i \alpha t} \in L^{2}\left(\mathbf{T}^{N}\right)$, that

$$
W f=W \sum a_{\alpha} S_{1}^{\alpha_{N}} 1, \ldots, S_{N}^{\alpha_{N_{1}}}=\sum a_{\alpha} e^{\imath \alpha t} W 1=\varphi f .
$$

Thus $W$ is multiplication by $\varphi$ and $\varphi$ is unimodular since $W$ is unitary.

Now given two invariant subspaces $\mathscr{M}_{1}$ and $\mathscr{M}_{2}$ which are unitarily equivalent, there are several possibilities for the function $\varphi$ of Lemma 1:

(a) $\varphi$ is constant;

(b) $\varphi$ is inner;

(c) $\varphi$ is the quotient of relatively prime inner functions;

(d) $\varphi$ is the quotient of inner functions; or

(e) $\varphi$ is not the quotient of inner functions.

When (a) holds, $\mathscr{M}_{1}=\mathscr{M}_{2}$; we obtain some results in this direction. Note that since, on $\mathbf{T}^{N}$, there exist nontrivial inner functions, i.e. nonconstant, unimodular functions in $H^{2}\left(\mathbf{T}^{N}\right)$, it follows that there exist distinct unitarily equivalent invariant subspaces. If $\mathscr{M}$ is an invariant subspace and $\varphi$ is an inner function, then $\varphi \mathscr{M} \subset \mathscr{M}$ with equality occurring if and only if $\varphi$ is constant; in fact $\varphi \mathscr{M} \subseteq \mathscr{M}$ if and only if $\varphi$ is inner. This is proved in Proposition 3 below. A consequence is that the unitary operator $U$ of Lemma 1 is unique up to a constant factor of modulus 1 . 
The statement that the inner functions $\varphi_{1}$ and $\varphi_{2}$ are relatively prime means that whenever

$$
\varphi_{1} f_{1}=\varphi_{2} f_{2}
$$

holds for $f_{1}, f_{2}$ in $H^{2}\left(\mathbf{T}^{N}\right)$, it follows that

$$
f_{2} / \varphi_{1}=f_{1} / \varphi_{2} \in H^{2}\left(\mathbf{T}^{N}\right) .
$$

If (c) holds then $\mathscr{M}_{1}$ and $\mathscr{M}_{2}$ can be represented as

$$
\mathscr{M}_{1}=\varphi_{1} \mathscr{M}, \quad \mathscr{M}_{2}=\varphi_{2} \mathscr{M},
$$

for the subspace

$$
\mathscr{M}=\left\{f / \varphi_{1}: f \in \mathscr{M}_{1}\right\}=\left\{f / \varphi_{2}: f \in \mathscr{M}_{2}\right\} .
$$

An apparently weaker condition is (d), where the inner functions $\varphi_{1}$ and $\varphi_{2}$ are not assumed to be relatively prime. In that case, the space $\mathscr{M}$ defined above is an invariant subspace of $L^{2}\left(\mathbf{T}^{N}\right)$, but not of $H^{2}\left(\mathbf{T}^{N}\right)$. Expressing $\mathscr{M}_{1}$ and $\mathscr{M}_{2}$ in terms of $\mathscr{M}$ as in (1), with $\varphi_{1}$ and $\varphi_{2}$ inner is equivalent to (c) if $\mathscr{M}$ can be taken to be an invariant subspace of $H^{2}\left(\mathbf{T}^{N}\right)$ or (d) if it is an invariant subspace of $L^{2}\left(\mathbf{T}^{N}\right)$. An interesting question is to give conditions under which an invariant subspace of $L^{2}\left(\mathbf{T}^{N}\right)$ is unitarily equivalent to an invariant subspace of $H^{2}\left(\mathbf{T}^{N}\right)$. The problem of whether an arbitrary unitarily equivalent pair of invariant subspaces has a representation (1) is equivalent to the following problem concerning the modulus of an $H^{2}\left(\mathbf{T}^{N}\right)$ function.

If $f_{1}$ and $f_{2}$ are functions in $H^{2}\left(\mathbf{T}^{N}\right)$ satisfying $\left|f_{1}\right|=\left|f_{2}\right|$ a.e. on $\mathbf{T}^{N}$, do there exist inner functions $\varphi_{1}$ and $\varphi_{2}$ such that $f_{1} \varphi_{2}=f_{2} \varphi_{1}$ ?

To see the equivalence in one direction, observe that if $\varphi \mathscr{M}_{1}=\mathscr{M}_{2}$ and if $f_{1}$ is a nonzero function in $\mathscr{M}_{1} \subset H^{2}\left(\mathbf{T}^{N}\right)$, then $\varphi f_{1}=f_{2}$ is a nonzero function in $\mathscr{M}_{2} \subset H^{2}\left(\mathbf{T}^{N}\right)$. Since $\varphi=f_{1} / f_{2}$ we see that if a representation as a quotient of inner functions is possible for $f_{1} / f_{2}$, then it is possible for $\varphi$. Conversely, if such a representation is not possible for $f_{1} / f_{2}$, then the cyclic invariant subspaces $\mathscr{M}_{1}$ and $\mathscr{M}_{2}$ generated by $f_{1}$ and $f_{2}$ are unitarily equivalent but have no representation of the form (1), since the function $\varphi$ given by Lemma 1 in this case is just $f_{2} / f_{1}$.

Added in proof. Case (d) (without (c)) and case (e) can, in fact, occur. Examples have recently been given by W. Rudin [J. Funct. Analysis, 61 (1985), 378-384].

2. We now set down two different sets of hypotheses under which we have made progress on concluding that unitarily equivalent invariant subspaces must be equal. The first is geometrical in nature, while the second is function theoretic. Both cover the case in which the invariant subspaces have finite codimension in $H^{2}\left(\mathbf{T}^{N}\right)$. 
An invariant subspace $\mathscr{M}$ of $l^{2}\left(\mathbf{Z}_{+}^{N}\right)$ has full range if, for $n=1,2, \ldots, N$, we have

$$
\bigvee_{j=1}^{\infty} S_{n}^{* j} \mathscr{M}=l^{2}\left(\mathbf{Z}_{+}^{n-1} \times \mathbf{Z} \times \mathbf{Z}_{+}^{N-n}\right)
$$

Note that for any subspace $\mathscr{M}$ of $l^{2}\left(\mathbf{Z}_{+}^{N}\right)$ and any $n$ we have inclusion of the left side of (2) in the right side.

Proposition 1. If $\mathscr{M}$ is a full range invariant subspace then any invariant subspace unitarily equivalent to $\mathscr{M}$ is of the form $\mathscr{N}=\varphi \mathscr{M}$, for some inner function $\varphi$.

Proof. In the notation of Lemma 1 , we have $\mathscr{N}=\varphi \mathscr{M}$, for some unimodular function $\varphi$. Thus we have

$$
M_{\varphi}\left(\bigvee_{j=1}^{\infty} S_{n}^{* j} \mathscr{M}\right)=\bigvee_{j=1}^{\infty} S_{n}^{* J} M_{\varphi} \mathscr{M}=\bigvee_{J=1}^{\infty} S_{n}^{* J} \mathscr{N}
$$

and since $\mathscr{M}$ has full range, it follows that

$$
M_{\varphi}\left(l^{2}\left(\mathbf{Z}_{+}^{n-1} \times \mathbf{Z} \times \mathbf{Z}_{+}^{N-n}\right)\right) \subset l^{2}\left(\mathbf{Z}_{+}^{n-1} \times \mathbf{Z} \times \mathbf{Z}^{N-n}\right) .
$$

Taking intersection over $n=1,2, \ldots, N$ gives

$$
M_{\varphi} l^{2}\left(\mathbf{Z}_{+}^{N}\right) \subset l^{2}\left(\mathbf{Z}_{+}^{N}\right)
$$

which implies that $\varphi=M_{\varphi} 1 \in H^{2}\left(\mathbf{T}^{N}\right)$, and hence is inner.

COROllary 1. An invariant subspace $\mathscr{M}$ is unitarily equivalent to $H^{2}\left(\mathbf{T}^{N}\right)$ if and only if $\mathscr{M}=\varphi H^{2}\left(\mathbf{T}^{N}\right)$ for some inner function $\varphi$.

CoRollary 2. If $\mathscr{M}_{1}$ and $\mathscr{M}_{2}$ are full range invariant subspaces, then they are unitarily equivalent if and only if they are equal.

COROLlaRy 3. If $\mathscr{M}_{1}$ and $\mathscr{M}_{2}$ are invariant subspaces of finite codimension in $H^{2}\left(\mathbf{T}^{N}\right)$, then they are unitarily equivalent if and only if they are equal.

Proof. Proposition 1 applies since a subspace of finite codimension has full range.

REMARK 1. This result should be compared with the situation in case $N=1$, in which all nonzero invariant subspaces of $H^{2}(\mathbf{T})$ are unitarily equivalent. 
REMARK 2. The result of Corollary 3 remains true if $H^{2}\left(\mathbf{T}^{N}\right)$ is replaced by an invariant subspace $\mathscr{M}$ of $H^{2}\left(\mathbf{T}^{N}\right)$ (see Proposition 4 below).

REMARK 3. Let $\left\{\varphi_{k}\right\}$ be a sequence of inner functions on $\mathbf{T}$ satisfying $\varphi_{k} H^{2} \subset \varphi_{k+1} H^{2}$ for $k=0,1,2, \ldots$ and

$$
\bigvee_{k=0}^{\infty} \varphi_{k} H^{2}=H^{2}
$$

If we let $\mathscr{M}$ be the subspace

$$
\mathscr{M}=\bigvee_{k=0}^{\infty} e^{i k t_{1}} \varphi_{k}\left(e^{i t_{2}}\right) H^{2}\left(\mathbf{T}^{2}\right),
$$

then $\mathscr{M}$ is a full range invariant subspace which has infinite codimension unless some $\varphi_{k}$ is a constant. If $\left\{\psi_{k}\right\}$ is another sequence of inner functions on $\mathbf{T}$ having the same properties, then the span of the doubly infinite sequence of subspaces

$$
\left\{e^{\imath\left(k_{1} t_{1}+k_{2} t_{2}\right)} \varphi_{k_{1}}\left(e^{l t_{2}}\right) \psi_{k_{2}}\left(e^{i t_{1}}\right) H^{2}\left(\mathbf{T}^{2}\right)\right\}
$$

is a more complex example of the same phenomenon.

If $\mathscr{N}$ is an invariant subspace of $H^{2}\left(\mathbf{T}^{2}\right)$ and $\varphi\left(e^{i t_{1}}, e^{i t_{2}}\right)$ is a nonconstant inner function, then $\varphi \mathscr{N}$ is an invariant subspace not having full range. It would be interesting to know if every invariant subspace not having full range can be written in this way.

3. Our function theoretic approach depends upon knowing that certain kinds of functions lie in the subspaces.

Proposition 2. Let $\mathscr{M}$ be an invariant subspace such that, for each $n$, $1 \leq n \leq N, \mathscr{M}$ contains a function independent of $e^{i t_{n}}$. Then every invariant subspace unitarily equivalent to $\mathscr{M}$ is of the form $\varphi \mathscr{M}$, for some inner function $\varphi$.

Proof. With $\varphi$ as in Lemma 1, write

$$
\varphi \sim \sum_{j=-\infty}^{\infty} f_{j}\left(e^{i t_{2}}, e^{i t_{3}}, \ldots, e^{i t_{N}}\right) e^{i j t_{1}} .
$$

If $p$ is a nonzero function in $\mathscr{M}$ which is independent of $t_{1}$, we have that $\varphi p$ is in $H^{2}\left(\mathbf{T}^{N}\right)$. Since $\varphi p$ has the expansion

$$
\varphi p \sim \sum_{j=-\infty}^{\infty}\left(p f_{j}\right) e^{i j t_{1}}
$$


this implies $p f_{j}=0$ for $j<0$ and hence $f_{j}=0$ for $j<0$. Treating the expansions of $\varphi$ relative to the other variables $e^{i t_{n}}, n=2,3, \ldots, N$, shows that $\varphi$ is in $H^{2}\left(\mathbf{T}^{N}\right)$ and hence is inner.

Corollary 4. If $\mathscr{M}_{1}$ and $\mathscr{M}_{2}$ are invariant subspaces, both of which contain functions independent of $e^{i t_{n}}$, for $n=1,2, \ldots, N$, then $\mathscr{M}_{1}$ and $\mathscr{M}_{2}$ are unitarily equivalent if and only if they are equal.

Remark 4. Of course, Corollaries 1 and 3 can also be derived from Proposition 2; the latter because if $\mathscr{M}$ has finite codimension in $H^{2}\left(\mathbf{T}^{N}\right)$, then $\mathscr{M}$ always contains the functions

$$
\left(e^{i t_{1}}-z_{1}\right)^{k},\left(e^{i t_{2}}-z_{2}\right)^{k}, \ldots,\left(e^{i t_{N}}-z_{N}\right)^{k}
$$

for some $\left(z_{1}, z_{2}, \ldots, z_{N}\right) \in \mathbf{D}^{N}$ and a sufficiently large integer $k$; [2, Theorem 3].

4. Carrying our polydisk function theory ideas a little further allows us to obtain the promised result on inclusion of invariant subspaces.

Proposition 3. Two invariant subspaces $\mathscr{M}_{1}$ and $\mathscr{M}_{2}$ satisfying $\mathscr{M}_{2} \subseteq$ $\mathscr{M}_{1}$ are unitarily equivalent if and only if $\mathscr{M}_{2}=\varphi \mathscr{M}_{1}$, for some inner function $\varphi$.

Proof. Unitary equivalence of $\mathscr{M}_{1}$ and $\varphi \mathscr{M}_{1}$ is obvious. Conversely, if $\mathscr{M}_{1}$ and $\mathscr{M}_{2}$ are unitarily equivalent, $\varphi$ is the unimodular function given by Lemma 1 , and $f$ is a nonzero function in $\mathscr{M}_{1}$, then $\varphi^{k} f$ is in $H^{2}\left(\mathbf{T}^{N}\right)$ for $k=1,2, \ldots$ Using an idea of Schneider [12], we shall prove this implies $\varphi \in H^{\infty}\left(\mathbf{T}^{N}\right)$.

We set $g=\varphi f$ and extend $\varphi$ to those $z$ in $\mathbf{D}^{N}$ where $f(z) \neq 0$ by defining

$$
\varphi(z)=g(z) / f(z) .
$$

To prove the extended function $\varphi$ is bounded and holomorphic in $\mathbf{D}^{N}$, we first claim that $|\varphi(z)| \leq 1$ at any point $z$ in $\mathbf{D}^{N}$ where $f(z) \neq 0$.

To prove this claim, set $h_{k}=\varphi^{k} f$. Since

$$
h_{k}\left(e^{i t}\right)=\varphi\left(e^{i t}\right)^{k} f\left(e^{i t}\right)=g\left(e^{i t}\right)^{k} / f\left(e^{i t}\right)^{k-1},
$$

we have

$$
f\left(e^{i t}\right) h_{k}\left(e^{i t}\right)=\varphi\left(e^{i t}\right)^{k-1} f\left(e^{i t}\right) g\left(e^{i t}\right)=h_{k-1}\left(e^{i t}\right) g\left(e^{i t}\right) .
$$

Since $f, h_{k}, h_{k-1}$ and $g$ lie in $H^{2}\left(\mathbf{D}^{N}\right)$, both $f h_{k}$ and $h_{k-1} g$ are in $H^{1}\left(\mathbf{D}^{N}\right)$ and we have (by the Cauchy formula, for example) that for $z$ in $\mathbf{D}^{N}$,

$$
f(z) h_{k}(z)=h_{k-1}(z) g(z)
$$


or, by induction, that

$$
h_{k}(z)=g(z)^{k} / f(z)^{k-1}=\varphi(z)^{k} f(z)
$$

for $z$ in $\mathbf{D}^{N}, f(z) \neq 0$. Now since $\left|h_{k}\left(e^{i t}\right)\right|=\left|f\left(e^{i t}\right)\right|$ a.e. we have for every $z=\left(z_{1}, \ldots, z_{N}\right)$ in $\mathbf{D}^{N}$,

$$
\left|h_{k}(z)\right| \leq c\left\|h_{k}\right\|_{2}=c\|f\|_{2}
$$

where $c=\Pi_{1}^{N}\left(1-\left|z_{j}\right|^{2}\right)^{-1 / 2}$ and hence if $z$ is in $\mathbf{D}^{N}$ and $f(z) \neq 0$,

$$
|\varphi(z)|^{k} \leq c\|f\|_{2} /|f(z)| .
$$

Since the right hand side is independent of $k$, this proves $|\varphi(z)| \leq 1$.

Now the fact that $\varphi(z)=g(z) / f(z)$ is holomorphic in $\mathbf{D}^{N}$ follows from Hartogs' theorem. Thus $\varphi$ is holomorphic in $\mathbf{D}^{N}$, bounded in modulus by 1 and unimodular on $\mathbf{T}^{N}$, which proves that $\varphi$ is inner.

The next proposition gives the generalization of Corollary 3 referred to in Remark 2.

Proposition 4. If $\mathscr{M}_{1}$ and $\mathscr{M}_{2}$ are unitarily equivalent invariant subspaces contained in an invariant subspace $\mathscr{M}$ and if $\mathscr{M}_{1}$ has finite codimension in $\mathscr{M}$, then $\mathscr{M}_{2}=\varphi \mathscr{M}_{1}$, for some inner function $\varphi$.

Proof. As might be expected, the proof combines the ideas of the proofs of Propositions 2 and 3.

Let $\varphi$ be the unimodular function given by Lemma 1 . We claim that $\varphi^{k} f$ lies in $H^{2}\left(\mathbf{T}^{N}\right)$, for every $f$ in $\mathscr{M}$ and for $k=0,1,2, \ldots$

Of course the claim is correct if $k=0$. Now proceed by induction, assuming $\varphi^{k-1} f$ belongs to $H^{2}\left(\mathbf{T}^{N}\right)$, for every $f$ in $\mathscr{M}$. If $\mathscr{M}_{1}$ has codimension $m$ in $\mathscr{M}$, the functions $f, e^{i t_{1}} f, e^{i 2 t_{1}} f, \ldots, e^{i m t_{1}} f$ lie in $\mathscr{M}$ and so some linear combination $\sum c_{j} e^{i j t_{1}} f=p_{1}\left(e^{i t_{1}}\right) f$ belongs to $\mathscr{M}_{1}$. It follows that $\varphi p_{1}\left(e^{\imath t_{1}}\right) f$ lies in $\mathscr{M}_{2}$ and hence in $\mathscr{M}$, and therefore

$$
\varphi^{k} p_{1}\left(e^{i t_{1}}\right) f \in \varphi^{k-1} \mathscr{M} \subset H^{2}\left(\mathbf{T}^{N}\right),
$$

by the induction hypothesis. Repeating the argument with $t_{1}$ replaced by $t_{2}, t_{3}, \ldots, t_{N}$, we obtain $N$ polynomials $p_{1}\left(e^{i t_{1}}\right), p_{2}\left(e^{i t_{2}}\right), \ldots, p_{N}\left(e^{i t_{N}}\right)$ such that for $n=1,2, \ldots, N$,

$$
\varphi^{k} p_{n}\left(e^{i t_{n}}\right) f=p_{n}\left(e^{i t_{n}}\right) \varphi^{k} f
$$

belongs to $H^{2}\left(\mathbf{T}^{N}\right)$. The proof of Proposition 2 shows that this implies $\varphi^{k} f$ belongs to $H^{2}\left(\mathrm{~T}^{N}\right)$.

Now the proof of Proposition 3 shows that $\varphi$ is inner. 
5. We conclude this note by offering another proof that invariant subspaces defined by a certain class of finite codimensional ideals in $\mathbf{C}\left[z_{1}, z_{2}\right]$ have the property that they are unitarily equivalent if and only if they are equal. The methods used from complex geometry offer the possibility of a much more detailed analysis of these invariant subspaces than the results obtained above. This is the reason we include them here.

Recall that in [6] a certain class of commuting pairs of operators was studied. A pair $\mathscr{T}=\left(T_{1}, T_{2}\right)$ of bounded linear operators on the Hilbert space $\mathscr{H}$ is said to belong to the class $\mathscr{B}_{1}(\Omega)$, for $\Omega$ an open connected subset of $\mathbf{C}^{2}$, if the following conditions are satisfied:

(1) $\operatorname{ran} D_{\mathscr{T}-\lambda}$ is closed for $\lambda$ in $\Omega$, where

$$
D_{\mathscr{T}}: \mathscr{H} \rightarrow \mathscr{H} \oplus \mathscr{H}
$$

is defined by $D_{\mathscr{T}} x=T_{1} x \oplus T_{2} x$ and $\operatorname{ran} D_{\mathscr{T}}$ is the range of $D_{\mathscr{T}}$;

(2) $\bigvee_{\lambda \in \Omega} \operatorname{ker} D_{\mathscr{T}-\lambda}=\mathscr{H}$; and

(3) $\operatorname{dim} \operatorname{ker} D_{\mathscr{T}-\lambda}=1$, for $\lambda \in \Omega$.

To each $\mathscr{T}$ in $\mathscr{B}_{1}(\Omega)$ is associated the Hermitian holomorphic line bundle $E_{\mathscr{T}}$ defined by

$$
E_{\mathscr{T}}=\left\{(\lambda, x) \in \Omega \times \mathscr{H}: x \in \operatorname{ker} D_{\mathscr{T}-\lambda}\right\} .
$$

In [6] it is proved that the curvature of this bundle is a complete unitary invariant for the pair $\mathscr{T}$.

The relevance of this to the study of invariant subspaces $\mathscr{M}$ of $H^{2}\left(\mathbf{T}^{N}\right)$ is that the operator pairs $\left(S_{1}^{*}, S_{2}^{*}\right)$, where now $S_{1}$ and $S_{2}$ are the restrictions to $\mathscr{M}$ of multiplication by $e^{i t_{1}}$ and $e^{i t_{2}}$, respectively

$$
S_{J} f=e^{i t_{f}} f, \quad j=1,2 ; f \in \mathscr{M},
$$

belongs to $\mathscr{B}_{1}(\Omega)$ for some $\Omega \subset \mathbf{D}^{2}$ for many (and perhaps all) invariant subspaces $\mathscr{M}$. Thus if the curvature of the associated line bundle can be calculated, then one can decide when two invariant subspaces are unitarily equivalent.

Let $0 \leq p_{1}<p_{2}<\cdots<p_{r}$ and $0 \leq q_{r}<q_{r-1}<\cdots<q_{1}$ be integers and let $\left(\lambda_{1}, \lambda_{2}\right)$ be a point in $\mathbf{D}^{2}$. Let $\mathscr{I}_{p, q, \lambda}$ be the ideal in $\mathbf{C}\left[z_{1}, z_{2}\right]$ consisting of those polynomials $p(z)$ for which the Taylor series expansion

$$
p(z)=\sum \alpha_{j k}\left(z_{1}-\lambda_{1}\right)^{j}\left(z_{2}-\lambda_{2}\right)^{k},
$$

about the point $\left(\lambda_{1}, \lambda_{2}\right)$, has $\alpha_{j k}=0$ if $j \leq p_{l}$ and $k \leq q_{l}$ for some $1 \leq l \leq r$. The closure $\mathscr{M}_{p, q, \lambda}$ of $\mathscr{I}_{p, q, \lambda}$ in $H^{2}\left(\mathbf{T}^{2}\right)$ is an invariant subspace of finite codimension. Let $\mathscr{T}_{p, q, \lambda}$ denote the operator pair $\mathscr{T}=\left(S_{1}^{*}, S_{2}^{*}\right)$, 
defined above, for this invariant subspace. Another proof of the fact that the $\mathscr{M}_{p, q, \lambda}$ are nonunitarily equivalent, for distinct $p, q, \lambda$, can be based on the following

Proposition 5. The pair $\mathscr{T}_{p, q, \lambda}$ is in $\mathscr{B}_{1}\left(\mathbf{D}^{2} \backslash\{\lambda\}\right)$ and the curvature $\mathscr{K}_{p, q, \lambda}$ for the associated line bundle is given by

$$
\begin{aligned}
& \mathscr{K}_{p, q, \lambda}(\omega, \bar{\omega}) \\
& =\sum_{j, k=1}^{2}\left(\frac{\partial^{2}}{\partial \omega_{j} \partial \bar{\omega}_{k}} \log \frac{1}{\left(1-\left|\omega_{1}\right|^{2}\right)\left(1-\left|\omega_{2}\right|^{2}\right)}+\frac{\partial^{2}}{\partial \omega_{j} \partial \bar{\omega}_{k}} F_{p, q, \lambda}\right) d \omega_{j} \wedge d \bar{\omega}_{k},
\end{aligned}
$$

where

$$
\begin{aligned}
F_{p, q, \lambda}(\omega, \bar{\omega})= & \sum_{l=1}^{r+1}\left|\frac{\omega_{1}-\lambda_{1}}{1-\bar{\lambda}_{1} \omega_{1}}\right|^{2 p_{l-1}+2}\left|\frac{\omega_{2}-\lambda_{2}}{1-\bar{\lambda}_{2} \omega_{2}}\right|^{2 q_{l}+2} \\
& -\sum_{l=1}^{r}\left|\frac{\omega_{1}-\lambda_{1}}{1-\bar{\lambda}_{1} \omega_{1}}\right|^{2 p_{l}+2}\left|\frac{\omega_{2}-\lambda_{2}}{1-\bar{\lambda}_{2} \omega_{2}}\right|^{2 q_{l}+2}
\end{aligned}
$$

and $q_{r+1}=-1$.

Proof. One can reduce the proof of $\mathscr{T}_{p, q, \lambda} \in \mathscr{B}_{1}$ to the case $\left(\lambda_{1}, \lambda_{2}\right)=$ $(0,0)$ using a pair of Möbius transformations on $\mathbf{D}$ and then apply the result for this case proved in [7, p. 20]. The curvature calculation is done using the orthonormal basis

$$
\left\{\frac{\sqrt{1-\left|\lambda_{1}\right|^{2}} \sqrt{1-\left|\lambda_{2}\right|^{2}}\left(z_{1}-\lambda_{1}\right)^{k}\left(z_{2}-\lambda_{2}\right)^{l}}{\left(1-\bar{\lambda}_{1} z_{1}\right)^{k+1}\left(1-\bar{\lambda}_{2} z_{2}\right)^{l+1}}\right\}_{k, l=0}^{\infty}
$$

for $H^{2}\left(\mathbf{T}^{2}\right)$, a part of which forms a basis for $\mathscr{M}_{p, q, \lambda}$.

Corollary 5. The invariant subspaces $\mathscr{M}_{p, q, \alpha}$ and $\mathscr{M}_{r, s, \beta}$ are unitarily equivalent if and only if $p=r, q=s$ and $\left(\alpha_{1}, \alpha_{2}\right)=\left(\beta_{1}, \beta_{2}\right)$.

Proof. By definition, $\mathscr{M}_{p, q, \alpha}$ and $\mathscr{M}_{r, s, \beta}$ are unitarily equivalent if and only if $\mathscr{T}_{p, q, \alpha}$ and $\mathscr{T}_{r, s, \beta}$ are. Since these operator pairs both belong to $\beta_{1}\left(\mathbf{D}^{2} \backslash\{\alpha, \beta\}\right)$, their unitary equivalence reduces to a comparison of curvatures. By inspection, both curvatures are real analytic functions which extend to an open neighborhood of the closed bidisk. Fixing one of the variables on the unit circle, it is possible, by comparing Taylor coefficients as in [1], to show that equality of the curvatures implies that $p=r, q=s$ and $\left(\alpha_{1}, \alpha_{2}\right)=\left(\beta_{1}, \beta_{2}\right)$. We will not go into details. 
As mentioned earlier, the curvature contains encoded all the data for the invariant subspace, up to unitary equivalence, and should prove useful in a more detailed study. We hope to return to this at some later date.

\section{REFERENCES}

[1] O. P. Agrawal, Invariant subspaces of shift operator for the quarter plane, PhD Thesis, SUNY Stony Brook, 1983.

[2] P. R. Ahern and D. N. Clark, Invariant subspaces and analytic continuation in several variables, J. Math. Mech., 19 (1970), 963-969.

[3] C. A. Berger, L. A. Coburn and A. Lebow, Representation and index theory for $C^{*}$-algebras generated by commuting isometries, J. Functional Analysis, 27 (1978), 51-99.

[4] A. Beurling, On two problems concerning linear transformations in Hilbert space, Acta Math., 81 (1949), 239-255.

[5] A. Brown and P. R. Halmos, Algebraic properties of Toeplitz operators, J. Reine Angew, Math., 213 (1963), 89-102.

[6] M. J. Cowen and R. G. Douglas, Complex geometry and operator theory, Acta Math., 141 (1978), 188-261.

[7] On operators possessing an open set of eigenvalues, Proc. Fejer-Riesz conference, Budapest, 1980.

[8] _ On moduli for invariant subspaces, in Invariant subspaces and other topics (Timisoara/Herculane, 1981) pp. 65-73, Operator Theory: Adv. Appl. 6, Birkhaüser, Basel-Boston, Mass., 1982.

[9] R. G. Douglas, On extending commutative semigroups of isometries, Bull. London Math. Soc., 1 (1969), 157-159.

[10] H. Helson, Lectures on Invariant Subspaces, Academic Press, New York, 1964.

[11] W. Rudin, Function Theory in Polydisks, Benjamin, New York, 1969.

[12] R. B. Schneider, Isometries of $H^{p}\left(U^{n}\right)$, Canad. J. Math., 25 (1973), 92-95.

Received May 2, 1984. The second and third authors were supported by NSF grants.

UNIVERSITY OF KANSAS

LAWRENCE, KS 66045

UNIVERSITY OF GEORGIA

ATHENS, GA 30602

AND

SUNY

STONY BROOK, NY 11794 



\section{PACIFIC JOURNAL OF MATHEMATICS EDITORS}

V. S. VARADARAJAN (Managing Editor)
University of California
Los Angeles, CA 90024
HEBERT CLEMENS
University of Utah
Salt Lake City, UT 84112
CHARLES R. DEPRIMA
California Institute of Technology
Pasadena, CA 91125

\section{R. FINN}

Stanford University

Stanford, CA 94305

HeRmanN FlaschKa

University of Arizona

Tucson, AZ 85721

RAMESH A. GANGOLli

University of Washington

Seattle, WA 98195

ROBION KIRBY

University of California

Berkeley, CA 94720

\section{C. MoOre}

University of California

Berkeley, CA 94720

H. SAMELSON

Stanford University

Stanford, CA 94305

HAROLD STARK

University of California, San Diego

La Jolla, CA 92093

ASSOCIATE EDITORS
R. ARENS
E. F. BECKENBACH
B. H. NeUmanN
F. WOLF
K. Yoshida (1906-1982)

\section{SUPPORTING INSTITUTIONS}

UNIVERSITY OF ARIZONA

UNIVERSITY OF BRITISH COLUMBIA

CALIFORNIA INSTITUTE OF TECHNOLOGY

UNIVERSITY OF CALIFORNIA

MONTANA STATE UNIVERSITY

UNIVERSITY OF NEVADA, RENO

NEW MEXICO STATE UNIVERSITY

OREGON STATE UNIVERSITY
UNIVERSITY OF OREGON

UNIVERSITY OF SOUTHERN CALIFORNIA

STANFORD UNIVERSITY

UNIVERSITY OF HAWAII

UNIVERSITY OF TOKYO

UNIVERSITY OF UTAH

WASHINGTON STATE UNIVERSITY

UNIVERSITY OF WASHINGTON 


\section{Pacific Journal of Mathematics}

\section{Vol. 121, No. $1 \quad$ November, 1986}

Om P. Agrawal, Douglas Napier Clark and Ronald George Douglas,

Invariant subspaces in the polydisk $\ldots \ldots \ldots \ldots \ldots \ldots \ldots \ldots \ldots \ldots$

Christoph Bandt and Gebreselassie Baraki, Metrically invariant measures

on locally homogeneous spaces and hyperspaces $\ldots \ldots \ldots \ldots \ldots \ldots \ldots$

Marcy Mason Barge, Horseshoe maps and inverse limits ..............29

Russell Gene Bilyeu, Robert Richard Kallman and Paul Weldon Lewis,

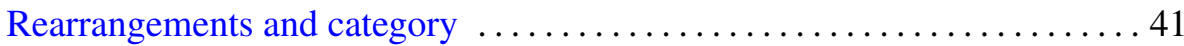

Jean Bourgain, A problem of Douglas and Rudin on factorization . . .......47

Hernan Cendra, A normal form and integration in finite terms for a class of

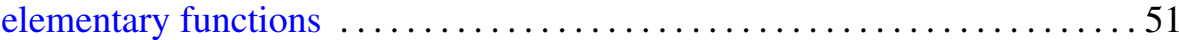

Ky Fan, The angular derivative of an operator-valued analytic function . . . . 67

Gerhard Gierz, On the Dunford-Pettis property of function modules of

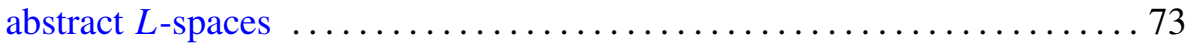

Gabriel Katz, On polynomial generators in the algebra of complex functions

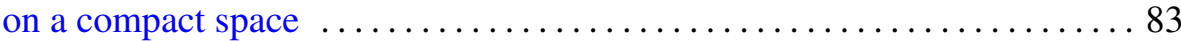

Ridgley Lange, Duality and asymptotic spectral decompositions $\ldots \ldots \ldots . .93$

Anthony To-Ming Lau and Peter F. Mah, Quasinormal structures for certain spaces of operators on a Hilbert space ................... 109

R. Daniel Mauldin, Correction: "The set of continuous nowhere differentiable functions"

Alan Harvey Mekler and Saharon Shelah, $\omega$-elongations and Crawley's problem

Alan Harvey Mekler and Saharon Shelah, The solution to Crawley's problem

Richard Rochberg, Deformation of uniform algebras on Riemann surfaces

Joseph Roitberg, On weak epimorphisms in homotopy theory

Jesús M. Ruiz, A remark on fields with the dense orbits property

Henry Wente, Counterexample to a conjecture of H. Hopf

David G. Wright, Rigid sets in $E^{n}$ 